\title{
A theoretical model for nutrient uptake in phytoplankton
}

\author{
D. L. Aksnes, J. K. Egge \\ Department of Fisheries and Marine Biology, University of Bergen, Thormohlensgt. 55, N-5008 Bergen, Norway
}

\begin{abstract}
A model for nutrient uptake rate $(V)$ in phytoplankton is derived: $V=n A v S /(1+h A v S)$ where $n$ is cellular number of uptake sites, $A$ is area of the uptake site, $h$ is time required for handling one nutrient ion, $v$ is the mass transfer coefficient, and $S$ is substrate concentration. The model is based on 2 time requirements necessary for active uptake of nutrients: (i) time required for realizing encounters with nutrient ions and (ii) time necessary for handling of ions. The Michaelis-Menten model applied to algal nutrient uptake is a special case of the proposed model, and shortcomings of this model are discussed in view of the more general model. While maximum uptake rate $\left(V_{\max }=n h^{-1}\right)$ may be considered as a parameter reflecting solely biological constraints, the half-saturation $\left(K_{\mathrm{s}}=(h A v)^{-1}\right)$ and affinity $(\alpha=\mathrm{n} A \mathrm{v})$ depend on both biological and physical constraints. Interrelations between maximum uptake rate, half-saturation constant and affinity are demonstrated and discussed with reference to actual observations. On the basis of the model we hypothesize that maximum uptake rate should increase linearly with the square of cell radius, while half-saturation constant and affinity should increase linearly with cell radius. Furthermore, maximum uptake rate should increase exponentially with temperature, while half-saturation should also increase with temperature, but at a slower rate than maximum uptake rate. Affinity should increase with temperature in the same way as molecular diffusion does.
\end{abstract}

\section{INTRODUCTION}

The kinetics of active transport through cell membranes is thought to resemble that of enzyme reactions (Lehninger 1971). This has also been demonstrated during the last several decades in experiments on nutrient uptake in phytoplankton. Accordingly, Michaelis-Menten kinetics has been the main descriptor for the uptake process. Originally, this approach was descriptive, but later the parameters maximum uptake rate $\left(V_{\max }\right)$ and half-saturation constant $\left(K_{\mathrm{s}}\right)$ were, to some extent, interpreted as estimates of theoretical parameters characterizing the species under study. Several experiments, however, have demonstrated shortcomings of using Michaelis-Menten kinetics on phytoplankton nutrient uptake. Droop (1983) wrote: The common and generally successful use of Michaelis kinetics to describe nutrient uptake tends to obscure the difficulties associated with interpretation of the kinetic parameters derived from the measurements as usually performed'. The interpretation of the half-saturation constant has been especially difficult. This parameter has often been considered to indicate competitive ability at low nutrient concentrations, and has sometimes been denoted the affinity constant. It was realized, however, that the observed covariation between $V_{\max }$ and $K_{\mathrm{s}}$ invalidated this interpretation (Law \& Button 1977). Instead the ratio $V_{\max } / K_{\mathrm{s}}(=\alpha)$ was suggested as a more appropriate affinity parameter (Button 1978, Healey 1980, Harrison et al. 1989).

Syrett (1956) reported increased nutrient uptake as a result of nutrient deficiency, and several later studies (Conway et al. 1976, McCarthy \& Goldman 1979, Goldman et al. 1981, Goldman \& Gilbert 1982, Parslow et al. 1984a, b. Harrison et al. 1989) have shown that nutrient-starved cells have an enhanced maximum uptake rate. This phenomenon is of course not encompassed by the 2-parameter Michaelis-Menten model, and Harrison et al. (1989) recommend that the maximum uptake should be determined both for starved cells $\left(V_{\max }\right)$ and for cells with a full nutrient pool $\left(V_{1}\right)$. Thus the time scale of experimental sampling was recognized as an important part of the nutrient uptake description. This was also pointed out by Collos (1980).

Maximum uptake rate, half-saturation constant and affinity are often said to be biological parameters characterizing the species under study. The physical 
aspects of nutrient uptake were, however, analysed and modelled (Munk \& Riley 1952) before MichaelisMenten kinetics came into common use. Munk \& Riley (1952) focused on the role of relative velocity between medium and individual cell and the consequences for potential nutrient utilization. Later, it was experimentally shown that the nutrient-depleted zone around individual cells is, due to transport limitation outside the cell, a potentially competitive factor at high cell densities (Hulburt 1970). Pasciak \& Gavis (1974, 1975) and Gavis (1976) combined the ideas of Munk \& Riley (1952) with the Michaelis-Menten approach to nutrient kinetics and found that transport limitation (outside the cell) is of significance for the realized nutrient uptake. Lazier \& Mann (1989) analysed influence of turbulence in water and cellular size on diffusion of substances to and from small spherical organisms. They conclude that high levels of turbulence in water have strong effect on the flux for organisms $>200 \mu \mathrm{m}$, but that the effect for smaller organisms is negligible.

The studies of Munk \& Riley (1952), Hulburt (1970), Pasciak \& Gavis $(1974,1975)$, Gavis (1976) and Lazier \& Mann (1989) show that the hydrodynamical environment is decisive for the realized uptake rate of nutrients in phytoplankton, and that the cells may adjust this environment by their size, shape and mobility. In the present paper we ask: How are such biological and environmental characteristics reflected in the parameters commonly estimated in uptake studies? To answer this question we have formulated a simple theoretical model where time requirements for nutrient uptake are divided into: (i) time necessary for encountering nutrient ions, and (ii) time necessary for handling ions. We demonstrate that the Michaelis-Menten model is a special case of the proposed model, and this allows for biological and physical interpretations of $V_{\max }, K_{\mathrm{s}}$ and $\alpha$. It is shown that $V_{\text {max }}$ reflects cellular morphology and intracellular processes while $K_{\mathrm{s}}$ reflects a composite of hydrodynamical processes, cellular morphology, mobility and intracellular processes. On the basis of our theoretical model, we suggest quantitative relationships between $V_{\max }, K_{\mathrm{s}}$ and $\alpha$. Our model is compared with models proposed by Munk \& Riley (1952), Caperon (1968) and Pasciak \& Gavis (1974). We calculate some order of magnitude estimates for the parameters in our model, and finally we propose 6 hypotheses that may be tested experimentally.

\section{THE MODEL}

\section{Time requirements of nutrient uptake}

Nutrient uptake refers to processes necessary for moving a nutrient (denoted as an ion) from the outside to the inside of a cell (or perhaps to a site where further processing of the ion takes place). This uptake is believed to be active, since passive diffusion can be refuted as a mechanism due to the higher nutrient concentrations found inside the algal cell. The rate of nutrient uptake depends on the time period needed for transfer of nutrient ions from the outside to the inside of the cell, but also on the time elapsing between encounters of nutrient ions and uptake sites. As the concentration of ions outside the cell decreases the encounter rate is likely to decrease correspondingly. Encounter rate, however, also depends on relative velocity between uptake sites and ions, and uptake rate may increase if this velocity is increased.

Neither biochemical nor hydrodynamical details will be considered in the present model. As a result, the fundamental assumptions of the model concern the 2 time requirements of the uptake process:

(i) Uptake of an ion requires an encounter (at or in the vicinity of the membrane) between the cell and the ion, and a certain number of encounters requires a finite time period.

(ii) The movement of a nutrient ion from the outside to the inside of the cell involves time-requiring handling of the ion.

Hence, our approach is similar to that of Holling (1959, 1966), modelling predation in animals. In his analysis too, prey encounter and prey handling are key processes. Our approach differs somewhat as the phytoplankton cell is allowed to have several 'mouths' (uptake sites). Consequently, one cell may encounter and handle many 'prey' (nutrient ions) simultaneously.

\section{Parameters and assumptions}

Uptake sites. We assume that the cell has a finite number of sites $(n)$ where encountered nutrient ions can be handled. Each site occupies a finite area $(A)$ of the membrane where ion catch is possible. Hence, the product $n A$ (which may vary) corresponds to the total area of the membrane where ion encounter may lead to uptake

Ion handling time $(h)$ is defined as the time interval where uptake of an ion at an uptake site is prevented because of handling of another ion.

Number of encountered ions $(m)$ at a site depends on substrate concentration (S), area of the uptake site (A), the time elapsed, and the mass transfer coefficient $(v)$. The mass transfer coefficient has the units $\mathrm{ms}^{-1}$ and is interpreted as the velocity ( $v$ ) between ions and uptake sites. It corresponds to the parameter denoted ' $h$ ' in Eq. (17) of Pasciak \& Gavis (1974). The mass transfer coefficient reflects mechanisms such as molecular diffusion and physical distortion of the 
medium governed by, for example, sinking, swimming (or just the activity of flagella), and turbulence in water (larger organisms). Due to the high viscosity at the scale of algal cells, these are likely to be surrounded with a layer of lowered nutrient content. Thus, a refined model requires parameterization of $S$ and $v$. This is not trivial, but an approximate formulation of $v$ has been used by Munk \& Riley (1952) and Pasciak \& Gavis (1974). Later, we will apply this approximation, but in the derivation of our model we just accept that $v$ may be measured in the unit $\mathrm{ms}^{-1}$ and represents a composite of physical and biological induced movements necessary for encounters between ions and cell membrane

\section{The equation}

As stated above each uptake site is considered to have a finite 'ion-catch' area $(A)$, and during a finite time interval $\left(T_{1}\right)$ one site will encounter $m$ ions:

$$
m=T_{1} A v S
$$

where $v=$ mass transfer coefficient; $S=$ nutrient ion concentration in the medium. As a result of ion handling, a site may be saturated for a period $(h)$, which precludes processing of other ions at that site. Total handling time required for processing $m$ encounters is denoted $T_{2}$, so that $T_{2}=m h$. Total time necessary for processing $m$ ions at one site is therefore $T=T_{1}+T_{2}$, and the uptake rate for one site ( $V^{\prime}$ ) becomes:

$$
V^{\prime}=m /\left(T_{1}+T_{2}\right)=\frac{A v S}{1+h A v S}
$$

If the cell has $n$ uptake sites the finite uptake rate $(V)$ for the cell is:

$$
V=\frac{n A v S}{1+h A v S}
$$

Handling and site limitation. From Eq. (3) it can be shown that cell uptake rate approaches a maximum rate, $n h^{-1}$, as the nutrient concentration $(S)$ increases. Accordingly, the uptake rate is limited by handling $(h)$ and number of sites per cell $(n)$ at high nutrient concentrations, and this may be interpreted as an exclusively biological limitation.

In the ecological situation handling time is seldom constant. Obviously, it depends on temperature as biochemical processes are involved. Furthermore, it is likely that the handling time depends on total nutrient uptake activity of the cell. This may be the case if handling depends on a common pool of energy or mass (as carriers). Then, an increased overall uptake activity may induce a carrier/energy limitation which will be reflected as increased handling time. Such increased handling would lead to decreased uptake rate (Eq. 3).
Encounter limitation. The uptake rate approaches $n A v S$ as the nutrient concentration decreases (Eq. 3). This quantity is the cellular encounter rate, and the cell is encounter limited at low nutrient concentrations. Such limitation is also termed transport (Pasciak \& Gavis 1974) and diffusion (Lazier \& Mann 1989) limitation. Encounter limitation is not solely an environmental physical constraint as biological characteristics such as number of sites $(n)$ and site area (A) also determine the encounter rate at low nutrient concentrations. Furthermore, as will be discussed later, the cell also influences encounter rate by size and mobility (though the parameters $v$ and $S$ ). Thus, encounter limitation should be interpreted as a combined physicalbiological limitation.

\section{Relative uptake rate and growth rate}

Eq. (3) gives the finite uptake rate for the cell, or the number of ions (of a specific element) entering a cell per unit time. For the sake of clarity we will also define the relative uptake rate, $V_{r}$. This is the number of elements extracted by the cell divided by the biomass (B) of the cell:

$$
V_{\mathrm{I}}=\frac{n A v S}{(1+h A v S) B}
$$

Biomass is assumed to be measured by the number of elements (of the same type considered in uptake) within the cell. Thus if nitrate uptake is measured, the number of nitrogen atoms would be the appropriate biomass unit. Normally, the number of elements $(B)$ within the cell doubles between cell divisions, but for simplicity, an average $B$ is assumed. Eq. (4) corresponds to the growth rate of the cell if only structural elements are part of the biomass quantity $B$ (i.e. the elements of the nutrient reservoir are excluded), if there is no lekage of elements from the cell, and if there is no change in the concentration of the internal nutrient pool. The unit of $V_{\mathrm{r}}$ is $\mathrm{s}^{-1}$, while the unit of $V$ (Eq 3 ) is number of nutrient ions $\mathrm{s}^{-1}$ cell $^{-1}$ (Table 1). In the comparison between our model and the growth model of Caperon (1968), Eq. (4) is used. Elsewhere, we refer to the finite rate ( $V$ ) expressed in Eq. (3).

\section{DISCUSSION}

The fundamental assumption of the proposed model is the division of time consumption into time needed for encounter and handling. These requirements can hardly be wrong although the time demands might be specified in much more detail by inclusion of hydrodynamical and biochemical mechanisms. Button (1985) 
Table 1. Explanation of symbols used in the text. $n_{\mathrm{s}}$ : number of sites; $n_{1}$ : number of nutrient ions

\begin{tabular}{|c|c|c|}
\hline Symbol & Definition & Unit \\
\hline A & Area of one uptake site & $\mathrm{m}^{2}$ \\
\hline$B$ & Cellular biomass & $n_{\mathrm{i}}$ \\
\hline$\alpha$ & Affinity & $\mathrm{m}^{3} \mathrm{~s}^{-1}$ cell- \\
\hline$D$ & Molecular diffusion & $m^{2} s^{-1}$ \\
\hline$E$ & Cellular surface are & $\mathrm{m}^{2}$ \\
\hline$g$ & $\ln \left(Q_{10}\right) / 10$ & ${ }^{\circ} \mathrm{C}^{-1}$ \\
\hline h & Handling time per ion & 5 \\
\hline$h_{0}$ & Handling time per ion at $0^{\circ} \mathrm{C}$ & s \\
\hline$K_{\mathrm{s}}$ & Half-saturation constant & $n_{\mathrm{i}} \mathrm{m}^{-3}$ \\
\hline$m$ & $\begin{array}{l}\text { Number of ions encountered } \\
\text { during time } T_{1} \text { at one site }\end{array}$ & $n_{\mathrm{i}}$ \\
\hline$n$ & Number of ion uptake sites per cell & $n_{\mathrm{s}}$ \\
\hline$n_{0}$ & Site density & $n_{\mathrm{s}} \mathrm{m}^{-2}$ \\
\hline Q:0 & $Q_{10}$ parameter for $h^{-1}$ & - \\
\hline$r$ & Radius of a spherical cell & $\mathrm{m}$ \\
\hline$S$ & $\begin{array}{l}\text { Concentration of nutrient ions in } \\
\text { the medium }\end{array}$ & $n_{2} \mathrm{~m}^{-3}$ \\
\hline$T_{1}$ & Time available for encounter & $\mathrm{s}$ \\
\hline$T_{2}$ & $\begin{array}{l}\text { Time spent processing ions } \\
\qquad(=m \times h)\end{array}$ & $\mathrm{s}$ \\
\hline$T$ & $\begin{array}{l}\text { Total time need to process } m \text { ions } \\
\qquad\left(=T_{1}+T_{2}\right)\end{array}$ & s \\
\hline$t$ & Temperature & ${ }^{\circ} \mathrm{C}$ \\
\hline$u$ & $\begin{array}{l}\text { Relative velocity between the cell } \\
\text { and the medium }\end{array}$ & $\mathrm{ms}^{-1}$ \\
\hline V & Ion uptake rate for the cell & $n_{1} \mathrm{~s}^{-1}$ \\
\hline$V^{\prime}$ & Ion uptake rate for one site & $n_{1} \mathrm{~s}^{-1}$ \\
\hline$V_{\max }$ & Maximum uptake rate for the cell & $n_{1} s^{-1}$ \\
\hline$V_{\mathrm{r}}$ & $=V / B$, relative uptake rate & $\mathrm{s}^{-1}$ \\
\hline $\mathrm{v}$ & Mass transfer coefficient & $\mathrm{m} \mathrm{s}^{-1}$ \\
\hline
\end{tabular}

reviews 4 different models for active transport mechanisms across the cell membrane, namely chemiosmotic, group translocation, ligand taxi and vectorial partioning. Although the biochemical mechanisms of these models are quite different, all mechanisms are characterized by time consumption that will be reflected in our handling parameter $(h)$. Inclusion of more mechanisms will certainly produce more parameters, but these are likely to be nested within the parameters already present in the proposed model. Therefore, we believe (or rather hope) that the overall structure of the model is non-susceptible to further reductionism. This is also supported by the fact that much of the experimental work carried out on nutrient uptake in phytoplankton is amazingly well described by the 2-parameter Michaelis-Menten model. In the following it is demonstrated that this model applied on nutrient uptake is a special case of our model. We do consider, however, important observations deviating from the Michaelis-Menten model, and suggest explanations in terms of the more general model. Thereafter, our model is compared to the models of Munk \& Riley (1952), Pasciak \& Gavies (1974) and Caperon (1968). Finally, we address the question of assessing experimentally derived parameter estimates and state some hypotheses that may be tested experimentally.

\section{The Michaelis-Menten model - a special case}

Rearrangement of Eq. (3) gives:

$$
V=\frac{n h^{-1} S}{(A v h)^{-1}+S}
$$

Hence, our model (Eqs. 3 and 5a) corresponds to the Michaelis-Menten model where:

$$
\begin{array}{ll}
V_{\max } & =n h^{-1} \quad \text { (maximum uptake rate) } \\
K_{\mathrm{s}} & =(A V h)^{-1} \quad \text { (half-saturation constant) }
\end{array}
$$

From Table 1 it may be ascertained that the units of $V_{\max }$ and $K_{\mathrm{s}}$ correspond to the units assessed in uptake experiments. According to our model, application of the Michaelis-Menten model to nutrient uptake relies on the assumption of a constant number of sites $(n)$, a constant handling time ( $h$ ), a constant site area $(A)$, and a constant mass transfer coefficient $(v)$. Thus, the validity of the Michaelis-Menten model depends on the time scale for possible alterations in these parameters.

Initial maximum uptake rate in starved cells. Several species that have been nutrient-starved for a certain period of time have exhibited a high initial maximum uptake rate on exposure to a supply of nutrients (Harrison et al. 1989). Thereafter maximum uptake decreases and approaches a constant level $\left(V_{1}\right)$, as the internal nutrient pool has been filled. Our model is in accordance with this phenomenon if either handling time $(h)$ increases or the number of sites $(n)$ decreases (or both) as the internal nutrient pool fills. As stated earlier, variable $h$ is reasonable if handling time depends on a common pool of energy or carriers. It is possible that a nutrient-poor cell has more carriers and energy allocated close to the membrane than a cell with a full nutrient reservoir. This may explain a short $h$ and thereby increased maximum uptake in starved cells.

The half-saturation constant $\left(K_{s}\right)$. According to Eq. $(5 c), K_{\mathrm{s}}$ decreases with increased area $(A)$ of the uptake site, increased mass transfer coefficient $(v)$, and increased handling time (h). Any ability of a cell to change the mass transfer coefficient (for example by sinking, swimming or altering size) should be reflected in the half-saturation parameter, but not necessarily in the maximum uptake rate.

The $K_{\mathrm{s}}$ value has often been interpreted as a measure of uptake efficiency at low nutrient concentrations where low $K_{\mathrm{s}}$ means high efficiency. Eq. (5c) may explain the inadequacy of this interpretation. Indeed, increase in site area $(A)$ and velocity $(V)$ increase 
uptake efficiency and such changes are appropriately reflected by a reduced $K_{\mathrm{s}}$ value. Decreased handling time, however, also increases uptake efficiency. According to Eq. (5c) such change is reflected as increased $K_{s}$ and this may incorrectly be interpreted as reduced nutrient-uptake ability at low substrate concentrations. As seen from Eq. (5b), shorter handling time also results in increased maximum uptake rate. Thus, the way $h$ influences both $V_{\max }$ and $K_{\mathrm{s}}$ leads to opposing effects on the realized uptake rate at low substrate concentrations. As shown later the appropriate affinity parameter $(\alpha)$ is actually independent of handling time. Thus, the present model gives support to the criticism of Healey (1980) and other authors on frequent misinterpretation of half-saturation constants in the literature.

By elimination of $h$ in Eqs. (5b) and (5c), a linear relationship between $V_{\max }$ and $K_{\mathrm{s}}$ is obtained:

$$
V_{\max }=n A v K_{\mathrm{s}}
$$

Such linear relationship is supported by the analysis of Collos \& Slawyk (1980) on experimental data on nitrate uptake extracted from several different sources.

Increased temperature generally means higher biochemical rates. Thus, it is reasonable that increased temperature will lead to shorter handling $(h)$ as such handling involves biochemical processes. The model predicts that shorter handling, caused by raised temperature, increases both $K_{\mathrm{s}}$ and $V_{\max }$ values, and this has been observed in several experiments (Eppley et al. 1969, Thomas \& Dodson 1974, Underhill 1977, Raimbault 1984).

The affinity, $\alpha$. Button (1978), Healey (1980) and Harrison et al. (1989) have suggested the ratio $\alpha=$ $V_{\max } / K_{5}$ as a more appropriate indicator of uptake ability at low nutrient concentrations. Button (1978) termed this parameter the affinity. According to our model the affinity is:

$$
\alpha=V_{\max } / K_{\mathrm{s}}=n A V
$$

which means that increase in number of uptake sites, their 'capture-area' and mass transfer coefficient facilitate increased affinity and thereby increased uptake rate under encounter limitation (i.e. at low nutrient concentrations). It should be noted that the handling time $(h)$ is not influencing $\alpha$ (Eq. 6). Nevertheless, as with $K_{s}, V_{\max }$ is coupled to $\alpha$, though now by the common parameter $n$ (number of sites). A combination of Eqs. (5b) and (6) gives:

$$
\alpha=h A v V_{\max }
$$

Note that $h$ in Eq. (7) is eliminated by the $h^{-1}$ occurring in $V_{\max }$ (Eq. 5b).

From Eqs. (5b) and (6) (or $5 b$ and 7) we see that increase in number of sites results in simultaneous increases in $V_{\max }$ and $\alpha$. The $\alpha$ may be interpreted as the sum of the total affinity of all sites (cellular affinity), where $A V$ is the site specific affinity. The unit $\left(\mathrm{m}^{3} \mathrm{~s}^{-1}\right)$ of affinity corresponds to ion clearance or 'filtration' rate.

\section{Present model in relation to models of Munk \& Riley (1952) and Pasciak \& Gavis (1974)}

Munk \& Riley (1952) derived formulas for the rate of nutrient absorption by aquatic plants depending on algal shape, size, sinking, substrate concentration and physical properties affecting transfer of nutrients toward the plant surface. Pasciak \& Gavis $(1974,1975)$ and Gavis (1976) extended the analysis to include empirical Michaelis-Menten uptake kinetics. Thus, they considered $V_{\max }$ and $K_{s}$ as organism-specific biological parameters. Our analysis suggests that $V_{\max }$ $\left(=n h^{-1}\right)$ may be interpreted as an exclusively biological parameter as $n$ is the number of uptake sites and $h$ reflects the time necessary for processing ions at or within the cell. We interpret the half-saturation constant, however, as a complex parameter reflecting both biological and physical mechanisms. Contrary to the model of Pasciak \& Gavis $(1974,1975)$ and Gavis (1976) our mass transfer coefficient $(v)$ is part of the halfsaturation parameter, and $V$ corresponds to ' $h$ ' in Eq. (17) of Pasciak \& Davis (1974). Their formulation of the mass transfer coefficient is based on an approximation used by Munk \& Riley (1952), but which was originally derived by Kronig \& Bruijsten (1951). In our notation, the mass transfer coefficient may be expressed:

$$
V=D / I+u / 2
$$

where $D=$ molecular diffusion; $r=$ cell radius; and $u=$ relative velocity between cell and medium. Phytoplankton sinking and swimming will be reflected in $u$. It should be noted that Eq. (8) is an approximation (for spherical cells) not valid for all phytoplankton motion. A detailed treatment of the validity is given by Gavis (1976). Turbulence in water should also be considered and included in the mass transfer coefficient for organisms larger than about $100 \mu \mathrm{m}$ (Lazier \& Mann 1989).

By using Eqs. (5c), (6) and (8):

$$
\begin{gathered}
K_{\mathrm{s}}=(A h[D / r+u / 2]\}^{-1} \\
\alpha=n A(D / r+u / 2)
\end{gathered}
$$

Hence, both the half-saturation parameter and the affinity are reflecting a composite of morphology ( $n$, number of sites; $A$, the catch area of a site; $r$, size of the cell), molecular diffusion (D) and mobility of the cell relative to the medium $(u)$. The half-saturation para- 
meter also incorporates handling time (h) which reflects the time requirement of internal cellular processes.

\section{Population growth model of Caperon (1968)}

Our model has much in common with the model of Caperon (1968) on population growth in micro-organisms limited by food supply:

$$
(1 / N)(\mathrm{d} N / \mathrm{d} t)=\left(k_{3} q^{-1} c_{0} N^{-1}\right) b /\left(b+k_{3} / k_{1}\right)
$$

This model expresses the specific growth rate of the population, where $N=$ number of individuals; $b=$ food or prey concentration; and $\mathrm{q}=$ the amount of food required to produce a new individual. According to Caperon (1968), $k_{1}$ may be looked upon as a measure of the effective volume swept by the predator, and $k_{3}$ is seen as a measure of the biochemical capability for relieving an occupied adsorption site, but is also related to chewing, swallowing or engulfing. The number of food-adsorption sites per individual is represented by $c_{0} N^{-1}$.

If the following substitutions are applied to Eq. (4): $A v=k_{1}, h=k_{3}{ }^{-1}, B=q, n=c_{0} N^{-1}$, and $S=b$, the relative uptake rate $V_{\mathrm{r}}$ (Eq. 4), becomes identical to Caperon's specific growth rate expressed in Eq. (10). According to Caperon (1968) an organism can increase the rate of food uptake from the environment in 3 ways:

(a) An increase in the effective size of the adsorption site (increase in $k_{1}$-our $A$ ).

(b) An increase in the number of adsorption sites per individuals (increase in $C_{0} / N$ - our $n$ ).

(c) An increase in the rate at which an occupied site is relieved (increase in $k_{3}$ - our $h^{-1}$ ).

According to our model there exists a fourth way to increase nutrient uptake:

(d) An increase in the mass transfer coefficient (increase in $v$ ).

This coefficient is influenced by the physical characteristics of the medium (through $D$ ), but is also controlled by the cell. According to Eq. (8), cell control is achieved by adjusting cell size $(r)$ or movement $(u)$. Decreased size and distortion of the medium at the scale of the algal cell will both increase $v$ and thereby increase the uptake rate.

Raven (1980) also lists 3 strategies to increase the influx of nutrients (our comments in parentheses):

(1) Greater density of porters (sites, $n$ ).

(2) Increased turnover (decreased $h$ ).

(3) Lower half-saturation parameter (lower $K_{s}$ ).

Strategies (1) and (2) correspond to (b) and (c) respectively, while Strategy (3) corresponds partly to (a), (b) and (d). This can be seen from our parameterization of the half-saturation parameter: $K_{\mathrm{s}}=(h A v)^{-1}$ where increase in handling time, site area and the mass transfer coefficient all make $K_{\mathrm{s}}$ smaller. It should be emphasized (as also stated above), however, that although increased handling time means decreased $K_{\mathrm{s}}$ such increase also means reduced $V_{\max }$ which implies reduced influx of nutrients. Thus, according to our model, Strategy (3) of Raven (1980) is not generally valid. If decrease in $K_{\mathrm{s}}$ is due to increased ion handling time, influx of nutrients will decrease rather than increase. On the other hand, if $K_{\mathrm{s}}$ decreases as a result of increased site area $(A)$ or increased mass transfer coefficient $(v)$, the influx of nutrients will increase as stated in (3) above.

\section{Parameter estimates}

The strength of the proposed model, as we see it, is that the parameters are biologically and physically interpretable. Unfortunately, this does not mean that they are easy to measure. The compatibility with the Michaelis-Menten model, however, is valuable. This compatibility means that measurements of uptake parameters obtained with current experimental methodology (Harrison et al. 1989) are relevant for the present model. Measurements of $V_{\max }, K_{\mathrm{s}}$ and $\alpha$ are actually estimates of the combined parameters $n h^{-1}$, $(A v h)^{-1}$ and $n A v$ respectively (Eqs. $5 b, 5 c$ and 6$)$. It should also be noted that the combined parameter $n A$ represents the area of the cell membrane which is capable of catching nutrient ions, and it is reasonable that this quantity is proportional to the cell surface. This indicates that the affinity $(\alpha=n A V)$ is proportional to the cell surface and thereby to the cell size. The velocity parameter $v$ is also related to cell size as well as morphology and mobility (Eq. 8). Thus, the present model may serve as a tool for quantitative integration of size, morphology and mobility on the one hand and the common uptake rate measurements on the other.

Despite the large number of published papers on estimates of nutrient uptake parameters, we have not been able to derive accurate estimates of the parameters in our model. Such achievement seems to require information on the number of sites. In Table 2 , however, we have presented calculations on the order of magnitude of the nitrate-uptake parameters of a 'typical', but hypothetical, algal cell with radius $3 \mu \mathrm{m}$, a doubling time of $12 \mathrm{~h}$, and a half-saturation constant of $0.5 \mu \mathrm{M}$. By these assumptions we have calculated possible combinations of site area, number of sites, ion handling time and site density (Table 2).

Raven (1980) reports that the total porter density rarely exceeds 3 pmol $\mathrm{cm}^{-2}$ (corresponding to $1.8 \times$ 
Table 2. Assumed and predicted parameter values of a hypothetical spherical algal cell with radius $3 \mu \mathrm{m}$. The wetweight to nitrogen ratio is assumed to be 80 . Maximum uptake rate was obtained by assuming that the $\mathrm{N}$-content of the cell has to double between cell divisions. $n_{1}$ refers to number of nutrient ions or to number of nitrogen atoms. Underlined numbers represent values within the ranges for site density (termed porter density by Raven) and ion handling time (taken as the inverse of Raven's turnover time) given by Raven (1980) (see text)

\begin{tabular}{|c|c|c|c|c|}
\hline \multicolumn{5}{|c|}{ Assumed values } \\
\hline \multicolumn{3}{|c|}{ Radius $(r)$} & 3.00 & $\times 10^{-6} \mathrm{~m}$ \\
\hline \multicolumn{3}{|c|}{ Surface $\left(E=4 \pi r^{2}\right)$} & 1.13 & $\times 10^{-10} \mathrm{~m}^{2}$ \\
\hline \multicolumn{3}{|c|}{ Biomass, wet-weight } & 1.16 & $\times 10^{-10} \mathrm{~g}$ \\
\hline \multicolumn{3}{|c|}{ Biomass, $N$-content $(B)$} & 6.23 & $\times 10^{10} n_{1}$ \\
\hline \multicolumn{3}{|c|}{ Doubling time $(\tau)$} & 4.32 & $\times 10^{4}$ \\
\hline \multirow{2}{*}{\multicolumn{3}{|c|}{$\begin{array}{l}\text { Maximum uptake rate }\left(V_{\max }=B / \tau\right) \\
\text { Half-saturation constant }\left(K_{\mathrm{s}}=0.5 \mu \mathrm{M}\right)\end{array}$}} & 1.44 & $\times 10^{6} \quad n_{1} \mathrm{~s}^{-1}$ \\
\hline & & & 3.01 & $\times 10^{20} n_{1} \mathrm{~m}^{-3}$ \\
\hline \multicolumn{3}{|c|}{ Affinity $(\alpha)$} & 4.79 & $\times 10^{-15} \mathrm{~m}^{3} \mathrm{~s}^{-1}$ \\
\hline \multicolumn{3}{|c|}{ Molecular diffusion $(D)$} & 1.5 & $\times 10^{-9} \mathrm{~m}^{2} \mathrm{~s}^{-1}$ \\
\hline \multicolumn{3}{|c|}{ Mass transfer coefficient $(v \approx D / r)$} & 5.0 & $\times 10^{-4} \mathrm{~m} \mathrm{~s}^{-1}$ \\
\hline \multicolumn{5}{|c|}{ Predicted values } \\
\hline \multirow{2}{*}{$\begin{array}{c}\text { Site } \\
\text { density } \\
n_{0}=\left(n_{5} m^{-2}\right)\end{array}$} & $\begin{array}{l}\text { No. of } \\
\text { sites }\end{array}$ & \multirow{2}{*}{\multicolumn{2}{|c|}{$\begin{array}{l}\text { Ion handling } \\
\text { time } \\
h=n / V_{\max } \\
\text { (s) }\end{array}$}} & \multirow{2}{*}{$\begin{array}{l}\text { Individual } \\
\text { site } \\
A=\alpha /(v n) \\
\left(\mathrm{m}^{2}\right)\end{array}$} \\
\hline & $n_{\mathrm{s}}=E n_{\mathrm{o}}$ & & & \\
\hline $10^{17}$ & $1.13 \times 10^{7}$ & 7.85 & & $8.5 \times 10^{-19}$ \\
\hline $10^{16}$ & $1.13 \times 10^{6}$ & $7.85 \times 1$ & $10^{-1}$ & $8.5 \times 10^{-18}$ \\
\hline$\overline{10^{15}}$ & $\overline{1.13} \times 10^{5}$ & $\overline{7.85 \times 1}$ & $10^{-2}$ & $8.5 \times 10^{-17}$ \\
\hline $10^{14}$ & $1.13 \times 10^{4}$ & $7.85 \times 1$ & $10^{-3}$ & $8.5 \times 10^{-16}$ \\
\hline $10^{13}$ & $1.13 \times 10^{3}$ & $7.85 \times 1$ & $10^{-4}$ & $8.5 \times 10^{-15}$ \\
\hline Total site ar & $n A=\alpha / v)$ & & & $9.6 \times 10^{-12} \mathrm{~m}^{2}$ \\
\hline - in perce & tage of surfa & e area & & $8.5 \%$ \\
\hline
\end{tabular}

$10^{16} n_{\mathrm{S}} \mathrm{m}^{-2}$ ) and that primary active transport porters have specific reaction rates not exceeding $100 \mathrm{~s}^{-1}$ (corresponding to $h=1 / 100=10^{-2} \mathrm{~s}$ ). In Table 2 values for ion handling time and site density which lie within Raven's ranges are underlined. Corresponding calculated values (using the model) for site area and number of sites are also underlined, and the model predicts that number of sites per cell should be in the range $10^{4}$ to $10^{6}$, and that individual site area should be in the range $10^{-18}$ to $10^{-16} \mathrm{~m}^{2}$. The predicted total site area $(n A)$ of the hypothetical cell is fixed at $8.5 \%$ of the cell surface. This assumes that only molecular diffusion $(D)$ contributes to the mass transfer coefficient $(v)$, and the estimate of total site area will be lower if the cell has significant ability to distort its surrounding medium (through the parameter $u$ ). The ion 'capture' efficiency of the sites also influence the estimates given in Table 2 , and the present calculations assume $100 \%$ capture efficiency for ions encountering a site. The size of an $\mathrm{N}-\mathrm{O}$ bond in the nitrate ion may be approximated from the single bond covalent radii for $\mathrm{N}$ and $\mathrm{O}$ which are 0.7 and $0.66 \times 10^{-10} \mathrm{~m}$ respectively (Cotton \&
Wilkinson 1976). Our estimates of probable site areas (underlined values in Table 2) corresponds to a site radius in the range $10^{-9}$ to $10^{-8} \mathrm{~m}$. Thus our model predicts that the capture area of a site in our hypothetical cell is larger than the ion, and this seems reasonable.

\section{Hypotheses}

Finally, we state 6 hypotheses about how the common measured uptake parameters should be related to cell size and temperature:

(1) $V_{\max }$ increases linearly with the squared cell radius.

$V_{\max }$ is proportional to number of sites, and number of sites is likely to be related to the cell surface. For a spherical cell: $V_{\max }=n h^{-1}=\left(h^{-1} n_{0} 4 \pi\right) r^{2}$, where $n_{0}$ is site density

(2) $K_{\mathrm{s}}$ increases linearly with cell radius.

By combined Eqs. (5c) and (8) we obtain $K_{\mathrm{s}}=(A h)^{-1}$ $(D / r+u / 2)^{-1}$. In the case where $u / 2 \ll D / r$ (which is likely for organisms not able to distort the surrounding medium) we obtain $K_{\mathrm{s}}=(A h D)^{-1} r$.

(3) $\alpha$ increases linearly with cell radius.

This follows from Hypotheses (1) and (2) as $\alpha=V_{\max }$ ' $K_{\mathrm{s}}$. Still assuming spherical cell and $u / 2 \ll D / r$ we obtain $\alpha=\left(n_{0} A 4 \pi D\right) r$.

The following hypotheses assume that biochemical reaction rates determining ion handling time $(h)$ is exponentially (as expressed in the $Q_{10}$-concept) related to temperature:

$$
h^{-1}=h_{0}^{-1} \exp (g t)
$$

where $t=$ temperature $\left({ }^{\circ} \mathrm{C}\right) ; h_{0}=$ ion handling time at $0^{\circ} \mathrm{C}_{;}$and $g=\ln \left(Q_{10}\right) / 10$.

(4) $V_{\max }$ increases exponentially with temperature.

This is apparent from the combination of Eqs. (5b) and (11) which gives $V_{\max }=n h_{0}^{-1} \exp (g t)$.

(5) $K_{\mathrm{s}}$ increases with temperature, but the increase is slower than for $V_{\max }$.

$K_{\mathrm{s}}$ is influenced by temperature not only through handling time, but also through the temperaturedependent molecular diffusion $(D)$ (which acts through the mass transfer coefficient [ $v$ ] [Eq. 8]). Combination of Eqs. (5c), (8) and (11) gives $K_{\mathrm{s}}=\left(A h_{0} r D(t)\right)^{-1} \exp (g t)$ where $D(t)$ indicates the temperature dependence of molecular diffusion. Molecular diffusion is linearly related to the temperature in the Stokes-Einstein equa- 
tion, but as the temperature-dependent viscosity also influences diffusion, the overall influence ot temperature on molecular diffusion is not straightforward. As molecular diffusion increases with temperature, however, the temperature influence on $K_{\mathrm{s}}$ through handling (h) time and diffusion act in opposite directions. Thus, the half-saturation should be less influenced by temperature than the maximum uptake rate.

(6) $\alpha$ increases with temperature in the same way as molecular diffusion does.

This is apparant from a combination of Eqs. (6) and (8) which results in $\alpha=n A(D(t) / r-u / 2)$,

Acknowledgements. We thank Y Collos, P. J. Harrison, B. Heimdal, E. Paasche, N. Swanberg and T F. Thingstad for valuable suggestions and comments on drafts of the manuscript.

\section{LITERATURE CITED}

Button, D. K. (1978). On the theory of control of microbial growth kinetics by limiting nutrient concentration. Deep Sea Res. 25: 1163-1177

Button, D. K. (1985). Kinetics of nutrient-limited transport and microbial growth. Microbiol. Rev. 49: 270-297

Caperon, J. (1968). Population growth in micro-organisms limited by food supply. Ecology 48: 715-722

Collos, Y (1980). Transient situations in nitrate assimilation by marine diatoms. I. Changes in uptake parameters during nitrogen starvation. Limnol. Oceanogr 25: 1075-1081

Collos, Y., Slawyk, G. (1980). Nitrogen uptake and assimilation by marine phytoplankton. In: Falkowski, P. G. (ed.) Primary productivity in the sea. Plenum Publ Corp., New York, p. 195-211

Conway, H. L., Harrison, P. J., Davis, C. O. (1976). Marine diatoms grown in chemostats under silicate or ammonium limitation. II. Transient response of Skeletonema costatum. to a single addition of the limiting nutrient. Mar. Biol. 35: $187-199$

Cotton, F. A., Wilkinson, G. (1976). Basic inorganic chemistry John Wiley \& Sons, New York

Droop, M. R. (1983). 25 years of algal growth kinetics - a personal view. Botanica mar 26: 99-112

Eppley, R. W., Rogers, J. N., McCarthy, J. J. (1969). Halfsaturation constants for uptake of nitrate and ammonium by marine phytoplankton. Limnol. Oceanogr. 14: 912-920

Gavis, J. (1976). Munk and Riley revisited: nutrient diffusion transport and rates of phytoplankton growth. J mar Res. 34: $161-179$

Goldman, J. C., Gilbert, P. M. (1982). Comparative rapid ammonium uptake by four species of marine phytoplankton. Limnol. Oceanogr 27:814-827

Goldman, J. C., Taylor, C. D., Gilbert, P. M. (1981). Nonlinear time-course uptake of carbon and ammonium by marine phytoplankton. Mar. Ecol. Prog. Ser. 6: 137-148

This article was submitted to the editor
Harrison, P. J., Parslow, J. S., Conway, H. L. (1989). Determination of nutrient uptake kinetic parameters: a comparison of methods. Mar Ecol. Prog. Ser. 52: 301-312

Healey, F. P. (1980). Slope of Monod equation as an indicator of advantage in nutrient competition. Microbiol. Ecol. 5: 281-286

Holling, C. S (1959). Some characteristics of simple types of predation and parasitism. Can. Entomol. 91: 385-398

Holling, C. S. (1966). The functional response of invertebrate predators to prey density. Mem. Entomol. Soc. Can. 48 : $1-86$

Hulburt, E. M. (1970). Competition for nutrients by marine phytoplankton in oceanic, coastal, and estuarine regions. Ecology 51: 475-484

Kronig, R., Bruijsten, J. (1951). On the theory of heat and mass transfer from a sphere in a flowing medium at low values of Reynold number. Appl. Sci. Res. 2: 439-446

Law, A. T., Button, D. K. (1977). Multiple-carbon-sourcelimited growth kinetics of a marine coryneform bacterium. J. Bacteriol. 129: 115-123

Lazier, J. R. N. Mann, K. H. (1989). Turbulence and the diffusive layers around small organisms. Deep Sea Res. 36: $1721-1733$

Lehninger, A. L. (1971). Bioenergetics. Benjamin/Cummings, New York

McCarthy, J. J., Goldman, J. C. (1979). Nitrogenous nutrition of marine phytoplankton in nutrient-depleted waters. Science 203: 670-672

Munk, W H., Riley, G. A. (1952). Absorption of nutrients by aquatic plants. J. mar. Res. 11: 215-240

Parslow, J. S., Harrison, P. J., Thompson, P. A. (1984a). Use of a self-cleaning in-line filter to continuously monitor phytoplankton nutrient uptake rates. Can. J. Fish. Aquat. Sci. 41: $540-544$

Parslow, J. S., Harrison, P. J., Thompson, P. S. (1984b). Development of rapid ammonium uptake during starvation of batch and chemostat cultures of the marine diatom Thalassiosira pseudonana to ammonium nitrate, silicate or phosphate starvation. Mar. Biol, 83: 51-59

Pasciak, W. J., Gavis, J. (1974). Transport limitation of nutrient uptake in phytoplankton. Limnol. Oceanogr. 19: 881-888

Pasciak, W. J., Gavis, J. (1975). Transport limited nutrient uptake rates in Ditylum brightwellii. Limnol. Oceanogr. 20: 605-617

Raimbault, P. (1984). Influence of temperature on the transient response in nitrate uptake and reduction by four marine diatoms. J. exp. mar. Biol. Ecol. 84: 37-53

Raven, J. A. (1980). Nutrient transport in microalgae. Adv microb. Physiol. 21-47-226

Syrett, P. J. (1956). The assimilation of ammonia and nitrate by nitrogen-starved cells of Chlorella vulgaris. II. The assimilation of large quantities of nitrogen. Physiol. Plant 9: $19-27$

Thomas, W H., Dodson, A. N. (1974). Effect of interactions between temperature and nitrate supply on the cell-division rates of two marine phytoflagellates. Mar Biol. 24 213-217

Underhill, P. S. (1977). Nitrate uptake kinetics and clonal variability in the neritic diatom Biddulphia aurita. J. Phycol. 13: $170-176$

Manuscript first received: August 16, 1990

Accepted: November 5, 1990 